\title{
Multidrug Resistance of Gastric Cancer: The Mechanisms and Chinese Medicine Reversal Agents
}

This article was published in the following Dove Press journal:

Cancer Management and Research

\author{
Wen-jie Huang ${ }^{1} * *$ \\ Shuai Ruan',* \\ Fang Wen ${ }^{2}$ \\ Xiao-na Lu' \\ Su-ping $\mathrm{Gu}^{2}$ \\ Xiao-xue Chen' \\ Miao Liu (D) ${ }^{3}$ \\ Peng Shu' \\ 'Oncology Department, Affiliated \\ Hospital of Nanjing University of Chinese \\ Medicine, Nanjing, People's Republic of \\ China; ${ }^{2}$ First College of Clinical Medicine, \\ Nanjing University of Chinese Medicine, \\ Nanjing, People's Republic of China; \\ ${ }^{3}$ Department of Pathology, Brigham and \\ Women's Hospital, Harvard Medical \\ School, Boston, MA, USA \\ *These authors contributed equally to \\ this work
}

\begin{abstract}
Chemotherapy is the main clinical treatment method of gastric cancer. Multidrug resistance (MDR) has become a common phenomenon with the development of tumors, which alleviates the effect of chemotherapy and makes it difficult to break the bottleneck of survival rate of advanced gastric cancer. Therefore, the exploration of MDR reversal agents for gastric cancer is the focus and also the difficulty of current treatment. Currently, the researches on the mechanisms of drug resistance in gastric cancer have been continuously deepened, which reveal different pathways and targets of MDR, laying a solid foundation for studying reversal agents. As a kind of natural medicine, traditional Chinese medicine (TCM) owns the characteristics of low toxicity, high safety and effectiveness. It can inhibit the occurrence, growth and metastasis of tumors, and reverse MDR via multiple pathways and mechanisms, the pathological function of which has become a research hotspot in recent years. TCM reversers are mainly divided into Chinese medicine monomers, Chinese patent medicines, and Chinese herbal compounds. With certain quantity and advantage, TCM reversers for MDR play an important role in the clinical treatment and show great potential in gastric cancer.
\end{abstract}

Keywords: gastric cancer, multidrug resistance, MDR, mechanisms, traditional Chinese medicine, TCM, review

Gastric cancer is a malignant tumor originating from the gastric mucosal epithelium and one of the leading causes of tumor-caused death worldwide. As reported by the global cancer statistics in 2018, gastric cancer remains a globally important cancer, with more than 1,000,000 new cases diagnosed and 783,000 deaths, ranking the fifth most frequently diagnosed cancer and the third main cause of cancer death. ${ }^{1}$

Chemotherapy is the central treatment of postoperative and metastatic gastric cancer. Classic chemotherapy drugs include 5-fluorouracil (5-Fu), cisplatin (DDP), paclitaxel, and epirubicin, among others. However, the efficacy of chemotherapy is restricted, and the 5-year overall survival rate of gastric cancer is still low, at only about $27.4 \% .{ }^{2}$ In recent years, with the continuous update of chemotherapy drugs, the survival time of patients with advanced gastric cancer has been prolonged but it is still in a bottleneck period. ${ }^{3,4}$ It is largely attributed to the existence of multidrug resistance (MDR) of gastric cancer cells, which causes less sensitivity to chemotherapy. MDR is the result of the combined actions of multiple factors and pathways, accompanied with the emergence of tumors. ${ }^{5}$ The MDR mechanisms found in previous studies include: increased drug efflux and metabolic abnormalities, increased DNA damage repair, reduced apoptosis, modification or alteration of drug active target proteins, the presence of tumor stem cells, the transformation of epithelial cells to mesenchymal
Oncology Department, Affiliated Hospital of Nanjing University of Chinese Medicine, I 55 Hanzhong Road, Nanjing, Jiangsu Province, People's Republic of China

Email shupengsp@163.com

Miao Liu

Department of Pathology, Brigham and

Women's Hospital, Harvard Medical

School, Boston, MA, USA

Email mliu0@bwh.harvard.edu 
cells, as well as regulation of hypoxia and hypoxia-inducible factor- $1 \alpha$ and microRNAs, etc. ${ }^{6}$ Most studies on the reversal of drug resistance in gastric cancer have focused on chemotherapy drugs. Up to now, there have been a lot of research results, and some drugs have been used in clinic, but the effects have not been fully confirmed. ${ }^{7}$ Traditional Chinese medicine (TCM) is a type of natural medicine, with characteristics of low toxicity, as well as high safety and effectiveness. It exerts inhibitory effects on the onset and development of gastric cancer, as well as MDR, by multiple pathways and mechanisms. Nowadays, it has become a research hotspot.

\section{Mechanisms of MDR in Gastric Cancer}

The main mechanisms of gastric cancer MDR are shown in Figure 1.

\section{Cell Membrane Transporter Abnormality}

As a classic MDR mechanism, transmembrane protein overexpression enhances the resistance of tumor cells to multiple structural and functional chemotherapeutic drugs by reducing intracellular drug concentration and preventing drug's binding to intracellular targets. It is the most important mechanism of MDR. Overexpression of adenosine triphosphate-binding cassette membrane transporters ( $\mathrm{ABC}$ transporters) is a major factor in the production of MDR, whose common family proteins include p-glycoprotein (p-gp), multi-drug resistance associate protein (MRP) and breast cancer resistance protein (BCRP). ${ }^{8}$ These proteins act as drug excretion pumps by reducing the concentration of chemotherapeutic drugs in tumor cells to develop resistance to multiple anti-tumor drugs. ${ }^{9}$

\section{Increased DNA Damage Repair and Decreased Apoptosis}

Recently, DNA damage repair has been found to be a novel and pivotal mechanism for MDR. Most of the chemotherapeutics currently used in clinical practice erase tumor cells by DNA damage to induce cell apoptosis. ${ }^{10}$ Researches on MDR of gastric cancer caused by DNA damage repair focus on platinum compounds which act upon DNA structures, such as XRCC1 acting on the BER pathway and BRCA1 acting on the HR pathway. ${ }^{11,12}$

Apoptosis is another vital mechanism for chemotherapeutic drugs to kill tumor cells. Apoptosis resistance and apoptosis escape are two important reasons for the formation of MDR. Apoptosis-related factors such as p53, Bcl-2, C-myc, Bax, nuclear transcription factor- $\kappa \mathrm{B}(\mathrm{NF}-\kappa \mathrm{B})$, Caspase-3, Ras, Survivin, mitogen-activated protein kinase (MAPK), pro-apoptotic factor cytochrome C (Cyt-C), apoptosis-inducing factor (AIF) and tumor necrosis factor- $\alpha$ (TNF- $\alpha)$ are all involved in MDR of carcinomas. ${ }^{13}$

\section{Existence of Cancer Stem Cells}

Cancer stem cells (CSCs) are tumor-forming cells accounting for a small proportion (only about 1\%) of all cancerous cells. CSCs are in the G0 phase of the cell cycle and have the property of self-renewal, which can resist the toxic effects of chemotherapy drugs and sow seeds for tumor proliferation and recurrence. ${ }^{14}$ The high expression of $\mathrm{ABC}$ transporter in CSCs is an other mechanism

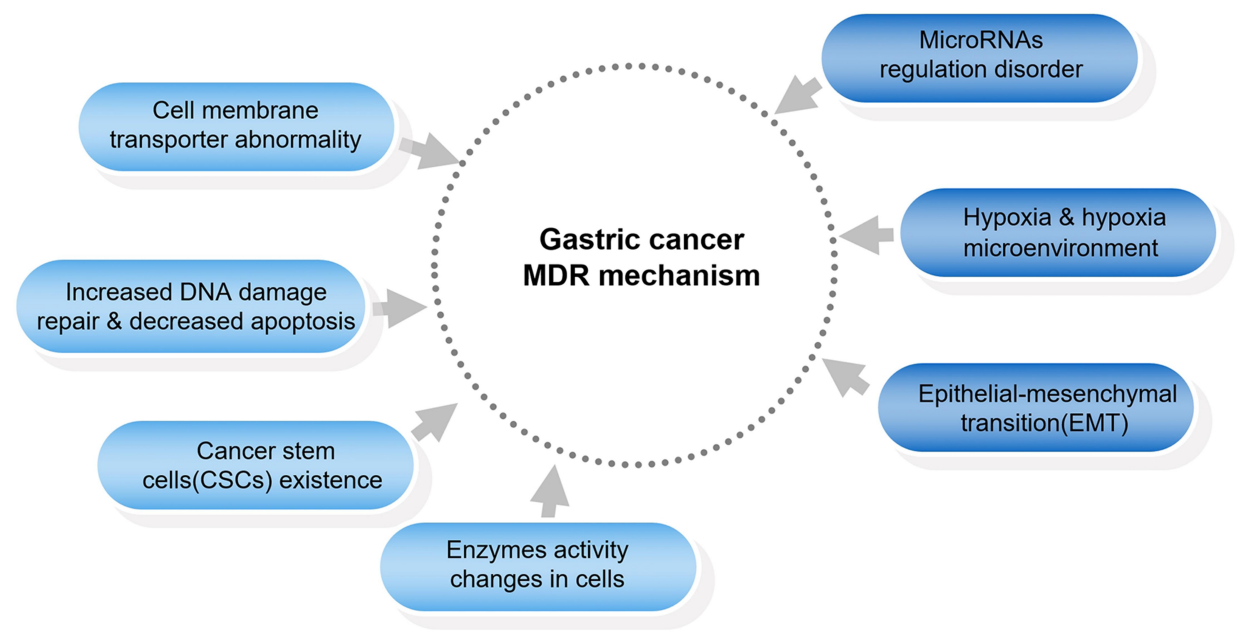

Figure I The main mechanisms of gastric cancer MDR. 
explicating the resistance to chemotherapy. ${ }^{15}$ Therefore, CSCs are not only the root of the tumor cells formation, but also a momentous cause of MDR in tumors.

\section{Changes in the Activity of Enzymes in Cells}

Compared with ordinary cells, specific changes in enzyme activity are observed in MDR cells, protein kinase C (PKC), topoisomerase II (Topo II), and glutathioneS-transferase- $\pi$ (GST- $\pi$ ) contained. PKC may lead to MDR formation by inducing MDR overexpression and accelerating p-gp phosphorylation. ${ }^{16}$ The decrease of Topo II in quantity and activity can result in drug resistance of tumor cells. GST- $\pi$ combined with lipophilic cytotoxic drugs can improve its water solubility and promote drug efflux, thus lessening the cytotoxic effect of anti-tumor drugs. ${ }^{17}$

\section{Epithelial-Mesenchymal Transition (EMT)}

EMT is closely related to the infiltration and distant metastasis of tumor cells, and thereafter, tumor cells acquire strong motility and invasiveness. In the study of oxaliplatin-resistant cell line SCG7901, drug-resistant cells develop EMT with overexpression of cellularmesenchymal to epithelial transition factor (c-MET). ${ }^{18}$ In addition, E-cadherin, a negative regulator of EMT, is absent in drug-resistant cells, but Vimentin is significantly increased, thereby facilitating the metastasis of drugresistant gastric cancer cells, which hints that EMT is in association with the formation of MDR in gastric cancer. ${ }^{19}$

\section{Hypoxia and the Formation of Hypoxia Microenvironment}

The formation of anoxic and anoxic microenvironment is one of the micro-environmental characteristics during solid tumor growth. Hypoxic microenvironment improves the growth, invasiveness and metastasis abilities of tumor tissues by affecting gene stability of tumor cells, upregulating glycolytic enzyme expression, down-regulating adhesion molecules, and maintaining stem cell characteristics, etc. ${ }^{20}$ Hypoxia can trigger MDR in plenty of solid tumors. ${ }^{21}$ In the study of gastric cancer, hypoxia significantly strengthens the resistance of SGC-7901 cells to five chemotherapeutics, including 5-fluorouracil (5-Fu), vincristine (VCR), cisplatin (DDP), etoposide (VP-16), and adriamycin (ADM). ${ }^{22}$ Clinical studies have presented that it makes the patients with gastric cancer resistant to platinum dugs and capecitabine chemotherapy, further proving its role as a considerable factor in the chemotherapy resistance of gastric cancer. ${ }^{23,24}$

\section{Disorder of MicroRNA Regulation}

MicroRNAs (miRNAs) are a growing and large family of short noncoding RNAs frequently dysregulated in malignancies. MiRNAs also play a key role in tumor chemotherapy resistance. ${ }^{25}$ There are many reports exploring the involvement of miRNAs in the MDR of gastric cancer, and the main pathways involved are as follows: ${ }^{26}$ regulating proteins related to drug resistance, such as miR-21, miR-27 and miR-129 by modulation of P-gp expression; influencing apoptosis, such as the regulation of Bcl-2 by miR-497, miR-181b and miR-204; accelerating EMT process like miR-1274a; inhibiting EMT process to reverse DDP resistance like miR-30. Changes in cell microenvironment and exosome forms partake in miRNAs related drug resistance as well.

\section{Chinese Medicine MDR Reversal Agents in Gastric Cancer Chinese Medicine Monomers}

Chinese medicine monomers refer to the compounds extracted from traditional Chinese medicinal materials, which is a substantial part of the active ingredients. It has a clear molecular structure, good biological activity and relatively distinct pharmacological action, which are of great application value for the research and development of MDR reversal agents. Therefore, Chinese medicine monomers are widely applied in treating tumor drug resistance. There are more than 20 monomers in the study of gastric cancer MDR.

\section{Curcumin}

Curcumin, a natural phenolic pigment, is widely found in Curcumae Longae Rhizoma, Curcumae Radix, and Curcumae Rhizoma, etc. Modern pharmacological studies have revealed that curcumin has broad-spectrum anticancer effects. Curcumin also develops an obvious reversal effect on gastric cancer MDR, and it can directly inhibit drug resistance of SGC7901 cells to ADR and VCR by reducing P-gp expression. ${ }^{27,28}$ In addition, several researches have shown that the reversal mechanism of curcumin for gastric cancer MDR is closely related with NF- $\kappa B$-mediated apoptosis. Kang and $\mathrm{Yu}$ found that curcumin can reverse 5-Fu, etoposide and doxorubicin resistance and hamper proliferation in gastric cancer cells by 
downregulating the NF- $\mathrm{KB}$ signaling pathway. The combination of curcumin and chemotherapeutics can induce apoptosis of SGC-7901 cells, attenuate the activation of $\mathrm{NF}-\mathrm{KB}$, and further lower the expression of NF$\kappa \mathrm{B}$-regulated anti-apoptotic genes like Bcl-2 and Bcl$\mathrm{xL}^{29,30}$ Moreover, Liu revealed that curcumin reverses trastuzumab resistance of NCI N87 cells possibly by inhibiting NF-KB pathway and activating cell apoptosis. In NCI N87/R cells, it preferentially hinders cell proliferation and NF- $\mathrm{kB}$ signaling pathway, downregulates the expression of HER-2 and Bcl-2, upregulates the expression of Bax, and reinforces the activity of Caspase- 3,8 and $9 .{ }^{31}$

\section{Tanshinone IIA}

Tanshinone IIA is one of the important fat-soluble monomer components harvested from Salviae Miltiorrhizae Radix et Rhizoma. It has been affirmed that tanshinone IIA possesses a wide range of anti-tumor activities through inhibiting cell growth, invasion, migration, and by potentiating apoptosis and differentiation. ${ }^{32} \mathrm{Liu}$ and $\mathrm{Xu}$ established two DOX-resistant cell lines SNU-719R and SNU-601R, discovering the suppressive effect of tanshinone IIA on the expression of MRP-1. Tanshinone IIA is an effective drug to inhibit the DOX resistance of gastric cancer by inducing cell cycle arrest. Combined with DOX, it can enhance apoptosis and provoke autophagic cell death, elevate the expressions of p53, Bax, LC3BII, and lower the expression of Bcl-2 and p62. ${ }^{33,34}$

\section{$\beta$-Elemene}

$\beta$-elemene is an anti-cancer active ingredient harvested from the Curcumae Radix, with strong anti-cancer activity in a variety of tumor treatments. It is a class I new-type anticancer Chinese medicine effectively reversing the MDR of tumors. $\beta$-elemene can mitigate drug resistance of SGC7901/ VCR to VCR and ADM, for which the decreases of P-gp and MRP may be responsible. ${ }^{35}$ And it alleviates the resistance and metastasis of exosome-mediated multidrug-resistant gastric cancer cell line SGC7901/ADR. ${ }^{36} \beta$-elemene also targets P-gp overexpressing gastric cancer cells SGC7901/ADR to enhance the efficacy of DOX treatment. The involved mechanisms contain the down-regulation of Akt phosphorylation, up-regulation of the E3 ubiquitin ligases, $\mathrm{c}-\mathrm{Cbl}$ and Cbl-b and activated fragment of PARP. ${ }^{37,38}$ In vivo, $\beta$ elemene significantly enhances the anti-tumor activity of DOX in nude mice bearing SGC7901/ADR xenografts and raises the expression of Caspase-3 protein. ${ }^{37,39}$

\section{Tetrandrine (TET)}

TET is an alkaloid isolated from the roots of Stephaniae Tetrandrae Radix. As a calcium antagonist, it is currently employed in the treatment of hypertension. TET has significant anti-tumor activity, which functions mainly by inducing pro-death apoptosis and autophagy in human gastric cancer cells. ${ }^{40}$ Studies have demonstrated that TET can down-regulate the mRNA and protein expression of ZNF139, MDR1, MRP1 and GST- $\pi$ in drug-resistant gastric cancer cells SGC7901/ ADR, suggesting its role in reversing MDR characteristics of gastric cancer. ${ }^{41,42}$

\section{Baicalein}

Baicalin is a flavonoid monomer compound isolated from the root of Scutellariae Radix. It is widely used owing to its anti-tumor, anti-inflammatory, and anti-virus effects. Baicalin can inhibit the invasion, migration and EMT of gastric cancer cells. ${ }^{43}$ Also, baicalein can reverse 5-Fu resistance in gastric cancer cells under hypoxia. It can downregulate the expression of MDR-related indicators (MDR1, ABCG2, MRPI) through HIF-1 $\alpha$ signaling pathway. ${ }^{44}$ Chen further found that the inhibition of glycolysis by regulating the PTEN/Akt/HIF-1 $\alpha$ signaling pathway may be one of the mechanisms by which baicalin improves 5 -Fu sensitivity in tumor cells under hypoxic conditions. ${ }^{45}$ In Hang's study, baicalin suppresses the growth and induces the apoptosis of SGC7901/DDP cells upon inhibition of Notch1 and upregulation of lncRNA AK022798. ${ }^{46}$

\section{$\mathrm{AS}_{2} \mathrm{O}_{3}$}

$\mathrm{AS}_{2} \mathrm{O}_{3}$ is the main component of Arsenolite, which can evoke apoptosis and differentiation, as well as inhibit the growth of various cancer cells. It can also repress MDR in gastric cancer mainly by increasing apoptosis, downregulating the expression of drug-resistant genes LRP, MRP and P-gp, and up-regulating the expression of apoptosis-related gene Caspase-3. ${ }^{47-49}$ Moreover, Zhao found that $\mathrm{AS}_{2} \mathrm{O}_{3}$ can reverse MDR of SGC7901/ADM by lowering the expression of P-gp in dose-dependent and time-dependent manners, which may be related to the participation of Ras/p-erk1/2 signaling pathway. ${ }^{50}$

\section{Other Chinese Medicine Monomers}

In addition to the above-mentioned reversal agents, there are more reversal agents of Chinese medicine monomers as listed in the Table 1. 
Table I Other Chinese Medicine Monomer Reversers

\begin{tabular}{|c|c|c|c|c|}
\hline Name & Target Genes/Proteins & Pathway & $\begin{array}{l}\text { Chemotherapeutic } \\
\text { Agent }\end{array}$ & Author \\
\hline Astragaloside-IV & MDRI, p-gP & - & DDP & Ye, $2017^{51}$ \\
\hline $\begin{array}{l}\text { Aqueous Extract of } \\
\text { Taxus Chinensis }\end{array}$ & Her-2 & PI3K/AKT & Trachuzumab & Wang, $2019^{52}$ \\
\hline Matrine & $\begin{array}{l}\text { mir-7, mir-125b, mir-200a, mir- } \\
200 b \text {, mir-200c, mir-146a }\end{array}$ & - & DDP & $\mathrm{Li}, 2015^{53}$ \\
\hline Neferine & $\mathrm{Bcl}-2$ & - & VCR & Shi, $2012^{54}$ \\
\hline Paeoniflorin & MDRI, Bcl-xl, Bcl-2 & $N F-\kappa B$ & VCR & Fang, $2012^{55}$ \\
\hline Bufalin & $\mathrm{Bax}, \mathrm{Bcl}-2$ & $\begin{array}{l}\text { Akt and the downstream } \\
\text { molecules-GSK-3ß, mTOR, S6K, } \\
\text { 4EBPI }\end{array}$ & DDP & $\begin{array}{l}\text { Zhang,2012 } 2^{56} \\
\text { Zhao,2016 }\end{array}$ \\
\hline Oleanolic acid & Bax, Bcl-2, Caspase-3 & - & DDP & $\mathrm{Li}, 2009^{58} \mathrm{Li}, 2010^{59}$ \\
\hline Avicularin & Bax, BOK, Caspase-3, PARP & - & DDP & Guo, $2018^{60}$ \\
\hline Peiminine & Cyclin DI, PARP & EGFR/FAK & ADR & Tang,2018 ${ }^{61}$ \\
\hline Tetramethylpyrazine & MDRI, GST-ח & - & ADR & Yang, $2015^{62}$ \\
\hline Sophoridine & B7-HI & - & DDP & Deng, $2013^{63}$ \\
\hline Triptolide & HSPAIA, 2HSPAIB/mir-2I, Bcl-2 & - & Apatinib/DDP & Teng,201 $8^{64}$ Long. $2012^{65}$ \\
\hline Gambogic acid & Surviving & - & Docetaxel & Wang, $2008^{66}$ \\
\hline
\end{tabular}

\section{Chinese Patent Medicines}

Chinese patent medicines are a kind of TCM products with certain dosage forms, which is made from several particular traditional Chinese medicinal materials under the guidance of TCM theory. Chinese patent medicines are often used as auxiliary drugs in tumor treatment for their stable curative effects and conveniences, especially among Chinese tumor patients. Although there are only few studies on the Chinese patent medicines in gastric cancer MDR, the existing studies have clarified the effectiveness of some drugs in resisting MDR of gastric cancer, which can provide a direction for future researches.

\section{Shengmai Injection}

Shengmai Injection is a TCM injection processed by modern pharmaceutical technology according to the classic prescription "Shengmai San". Its active ingredients mainly include ginsenoside, oligosaponin, schisandrin and the like. Previous studies have validated that Shengmai Injection possesses the effect of synergistic chemotherapy to increase the efficacy and reduce the side effects. ${ }^{67}$ Shengmai Injection combined with chemotherapy can restrict the growth of transplanted tumor by VCRresistant gastric cancer cells SGC7901 in nude mice, increase the lethality of chemotherapy drugs on gastric cancer cells, and accelerate tumor cell apoptosis. ${ }^{68}$ Furthermore, Shengmai Injection-contained serum blocks SGC7901/VCR cells in the G0/G1 phase, inhibits the proliferation, reduces the expression of MDR1 and MRP1, regulates the JNK signaling pathway by increasing c-jun phosphorylation, down-regulates the P-gp expression, and thus mitigates MDR. ${ }^{69}$

\section{Coix Seed Oil (Kanglaite ${ }^{\circledR}$ ) Injection}

Coix Seed Oil Injection is extracted and purified from Chinese herbal medicine Coicis Semen. It can inhibit the proliferation of tumor cells, kill tumor cells and enhance the immune function of the body ${ }^{70}$ Clinical researches have confirmed that Coix Seed Oil Injection strengthens the efficacy and reduces the side effects of gastrointestinal reactions and bone marrow suppression in chemotherapy, and improves the life quality of gastric cancer patients. ${ }^{71}$ Zhang confirmed the effect of Coix Seed Oil Injection in gastric cancer's MDR in which it restrains the cell viability and promotes the cell apoptosis of BGC823/DPP and SGC7901/DDP cells in a concentration-dependent manner. The repressive impact of Coix Seed Oil Injection on the expression of MDR1 and MRP1 via suppressing lncRNA PVT1 may be the potential mechanism. ${ }^{72}$

\section{Chinese Herbal Compounds}

Chinese herbal compounds are the most widely utilized medicine in the clinical treatment of cancer with TCM. According to the core principle of TCM treatment "syndrome differentiation and treatment" and the special pathological characteristics of the disease, the basic formula is 
composed of diverse traditional Chinese medicinal materials. Contraposing the characteristics of different patients, doctors add or delete several herbs to adjust the basic prescription during clinic application, reflecting the soul of TCM treatment.

\section{Jianpi Yangwei Decoction (Yiqi Huayu Jiedu Decoction)}

Jianpi Yangwei Decoction is composed of fifteen kinds of traditional Chinese medicinal materials, such as Astragali Radix, Codonopsis Radix, Poria, Angelicae Sinensis Radix, Sparganii Rhizoma, Curcumae Rhizoma. It emphasizes that strengthening the spleen and removing blood stasis are the two focuses of gastric cancer treatment. Shenliu Liu's team created this recipe and clinical researches confirmed that Jianpi Yangwei Decoction can reduce the risk of recurrence and metastasis in gastric cancer patients at stage II and III after surgery. The risk of recurrence and metastasis decreases by $32.8 \%$ compared with chemotherapy alone. ${ }^{73}$ The role of Jianpi Yangwei Decoction in alleviating gastric cancer MDR has also been confirmed by several reports. It triggers inactivation of the PI3K/AKT signaling pathway and inhibition of MDR1 expression in gastric cancer 5-Furesistance cells BGC823. ${ }^{74}$ In addition, Jianpi Yangwei Decoction also acts on tumor stem cells to reverse drug resistance. Tang found that it exerts anti-tumor effects by down-regulating the expression of Nanog, Oct-4 and Sox 2 proteins in BGC823/5-FuCD44 $(+)$ cells and promoting the differentiation of CSCs. ${ }^{75}$ Moreover, $\mathrm{Fu}$ found that it decreases the expression of MDR1, MRP1 and ABGC2 in BGC823/5-FuCD44 $(+)$ cells in a dose-dependent manner, thereby reversing $\mathrm{MDR}^{76}$

\section{Yiqi Jianpi Huaji Decoction}

The main herbs in Yiqi Jianpi Huaji Decoction are Astragali Radix, Codonopsis Radix, Atractylodis Macrocephalae Rhizoma, Paeoniae Radix Alba, Citri Reticulatae Pericarpium, Pinelliae Rhizoma, Salvia chinensis Benth, etc. Li revealed the effects and main mechanisms of this prescription in reversing gastric cancer MDR. Yiqi Jianpi Huaji Decoction undermines the proliferation of SGC7901/VCR, and its combination with 5-Fu increases apoptosis and blocks cells in $\mathrm{S}$ phase of cell cycle. The effects of Yiqi Jianpi Huaji Decoction may be attributed to the decrement of the expression of MDR1/ P-gp, MRP, TUBB3, and STMN1, thereby weakening the level of P-gp-mediated MDR and increasing the sensitivity of SGC7901/VCR cells to chemotherapy. ${ }^{77}$

\section{Shenghe Powder}

Shenghe Powder is made of mixed traditional Chinese medicinal material powders, such as Ginseng Radix et Rhizoma, Scrophulariae Radix, Coicis Semen, anemone and Bufonis Venenum. Wang's team has conducted the researches on the therapeutic effect of Shenghe Powder in gastric cancer for more than 20 years. Clinical studies have shown that Shenghe Powder combined with chemotherapy can reduce the recurrence and metastasis of gastric cancer after surgery. ${ }^{78}$ In addition, experimental studies have indicated that Shenghe Powder can reverse MDR of SGC7901/VCR. It increases the intracellular concentration of vincristine in SGC7901/VCR and promotes apoptosis, with the downregulation of P-gp and Bcl-2. The reversal effect of Shenghe Powder is stronger than that of Verapamil. ${ }^{79}$

\section{Banzhen I}

Banzhen 1 is a compound composed of four traditional Chinese medicinal materials, namely Mylabris, Polistes mandarinus Saussure, Ligustri Lucidi Fructus and Corni Fructus. Banzhen 1 combined with ligustrazine can inhibit the expression of LRP and MRP mRNA in SGC-7901/ ADR cell line and improve the drug sensitivity of SGC7901/ADR. ${ }^{80}$ The mechanisms of Banzhen 1 reversing MDR may be associated with enhanced apoptosis by upregulating Bax, as well as down-regulating Bcl-2 and Bcl2/Bax ratio. ${ }^{81}$ In addition, Banzhen 1 can also reduce GST$\pi$ expression in SGC-7901/ADR to reverse MDR. ${ }^{82}$

\section{Other Chinese Herbal Compounds}

Chang Wei Qing combined with chemotherapy can prolong the survival time and improve the life quality of patients with gastrointestinal tumors, as well as reduce the expressions of MDR1 mRNA and CK20 mRNA in patients' peripheral blood, which indicates that it has a synergistic effect on gastrointestinal cancer with chemotherapy through the reversal of MDR. ${ }^{83}$ Er Teng San Jie can alleviate the MDR by silencing the expression of P-pg in SGC7901/VCR to increase the concentration of intracellular $\mathrm{ADM}^{84}$ Sanwubai Powder is used as a potential drug to mitigate MDR by reducing the expression rate of MDR in SGC7901 cells. ${ }^{85}$

\section{Conclusion and Forecast}

MDR is a common phenomenon in the treatment of gastric cancer, which limits the effectiveness of chemotherapy. It 
is an important problem urgently to be solved. At present, the mechanisms of gastric cancer MDR have been investigated in depth. Modern scientific researches have explained the causes of gastric cancer MDR from multiperspectives and multi-dimensions, providing a foundation for the development of reversal agents. Currently, TCM, safe and effective, occupies a certain position in the research of reversing gastric cancer MDR, and has been applied in clinical practice to benefit patients.

However, it cannot be ignored that the mechanism of tumor MDR is complicated. In this study, we comprehensively collected and summarized the effects of Chinese medicine and Chinese medicine products in reducing the gastric cancer MDR in past 10 years, which preliminarily confirmed the effectiveness of Chinese medicine in reversing gastric cancer MDR. However, based on the current researches in this field, our research also has certain limitations. In terms of research objects, Chinese medicine MDR reversal agents mainly include Chinese medicine monomers, Chinese patent medicines, and Chinese herbal compounds. Among them, there is a higher proportion of studies on monomers, but a lower proportion of studies on Chinese patent medicines and herbal compounds, all of which are widely used in clinical practice. In terms of researches on the mechanisms of drug resistance, most of the current researches focus on the classical mechanism of MDR gene and P-gp encoded by MDR gene, while few works focus on non-classical mechanisms such as GST- $\pi$, Topo II, DNA damage repair and tumor stem cells, etc. By contrast, these mechanisms are scarcely explored and the research depth is relatively insufficient. In addition, more importantly, most of the current studies are experiments in vitro. We can observe that animal experiments in vivo have been performed in just a few studies, such as $\beta$-elemene, Shengmai Injection mentioned above. Moreover, there is a lack of clinical studies to a large extent. In this work, only the research of Chang Wei Qing detected drug resistance-related targets in peripheral blood of gastric cancer patients through clinical study. ${ }^{83}$ This phenomena are indeed the current research status of TCM in MDR. More in vivo and even clinical studies need to be conducted to deepen the current researches.

Based on the present researches, studies on pharmacology and metabolomics of Chinese medicine reversal agents can be launched, and on this basis, studies developing new Chinese medicine drugs for reversing MDR can be conducted, and large-sample randomized controlled clinical studies can be carried out to observe their actual effects in reversing drug resistance in gastric cancer patients during clinical treatment, providing a higher-level evidence for the therapeutic effects of TCM in gastric cancer MDR. We believe that the above problems will eventually be solved with the development of molecular biotechnology, gene proteomics, network pharmacology and other related disciplines. The mechanisms of TCM reversing MDR will be excavated more widely and deeply, and more reversal agents will be developed and applied in clinical practice.

\section{Abbreviations}

MDR, multidrug resistance; TCM, traditional Chinese medicine; 5-Fu, 5-fluorouracil; DDP, cisplatin; ABC transporters, adenosine triphosphate-binding cassette membrane transporters; P-gp, p-glycoprotein; MRP, multidrug resistance associate protein; BCRP, breast cancer resistance protein; $\mathrm{NF}-\kappa \mathrm{B}$, nuclear transcription factor$\kappa \mathrm{B}$; MAPK, mitogen-activated protein kinase; Cyt-C, proapoptotic factor cytochrome $\mathrm{C}$; AIF, apoptosis-inducing factor; TNF- $\alpha$, tumor necrosis factor- $\alpha$; CSCs, Cancer stem cells; PKC, protein kinase C; Topo II, topoisomerase II; GST- $\pi$, glutathione-S-transferase- $\pi$; EMT, epithelialmesenchymal transition; c-MET, cellular-mesenchymal to epithelial transition factor; VCR, vincristine; VP-16, etoposide; ADM, Adriamycin; miRNAs, MicroRNAs.

\section{Author Contributions}

All authors made a significant contribution to the work reported, whether that is in the conception, study design, execution, acquisition of data, analysis and interpretation, or in all these areas; took part in drafting, revising or critically reviewing the article; gave final approval of the version to be published; have agreed on the journal to which the article has been submitted; and agree to be accountable for all aspects of the work.

\section{Funding}

This research was supported by the National Natural Science Foundation of China (No. 81673918), the Open Projects of the Discipline of Chinese Medicine of Nanjing University of Chinese Medicine supported by the Subject of Academic Priority Discipline of Jiangsu Higher Education Institutions (No. ZYXO3KF020), the Pilot Gastric Cancer Project of Clinical Cooperation of Traditional Chinese and Western Medicine for Major and Difficult Diseases and the National Administration of Traditional Chinese Medicine; and 2019 Project of Building Evidence-Based Practice Capacity for TCM (2019XZZX-ZL003). 


\section{Disclosure}

The authors declare that they have no conflicts of interest.

\section{References}

1. Bray F, Ferlay J, Soerjomataram I, Siegel RL, Torre LA, Jemal A. Global cancer statistics 2018: GLOBOCAN estimates of incidence and mortality worldwide for 36 cancers in 185 countries. $C A$ Cancer J Clin. 2018;68(6):394-424. doi:10.3322/caac.21492

2. Zuo T, Zheng R, Zeng H, Zhang S, Chen W. Epidemiology of stomach cancer in China. Chin J Clin Oncol. 2017;44(1):52-58.

3. Song Z, Wu Y, Yang J, Yang D, Fang X. Progress in the treatment of advanced gastric cancer. Tumour Biol. 2017;39(7):101042831771 4626. doi: $10.1177 / 1010428317714626$

4. Ilson DH. Advances in the treatment of gastric cancer. Curr Opin Gastroenterol. 2018;34(6):465-468. doi:10.1097/MOG.0000000000 000475

5. Marin JJ, Al-Abdulla R, Lozano E, et al. Mechanisms of resistance to chemotherapy in gastric cancer anticancer agents. Med Chem. 2016;16(3):318-334.

6. Huang H, Yang X, Gao R. Research advances in the mechanisms of gastric cancer multidrug resistance. Zhongguo Yi Xue Ke Xue Yuan Хие Bao. 2016;38(6):739-745.

7. Li Y, Tan B. Current situation and trend of research for drug resistance in gastric cancer. World Chin J Digestol. 2016;24(32): 4330-4336. doi:10.11569/wcjd.v24.i32.4330

8. Shapira A, Livney YD, Broxterman HJ, Assaraf YG. Nanomedicine for targeted cancer therapy: towards the overcoming of drug resistance. Drug Resist Updat. 2011;14(3):150-163. doi:10.1016/j. drup.2011.01.003

9. Zhang $\mathrm{H}, \mathrm{Fu}$ L. Multidrug resistance-associated proteins and their roles in multidrug resistance. Acta Pharm Sin B. 2011;46(5):479-486.

10. Gavande NS, VanderVere-Carozza PS, Hinshaw HD, et al. DNA repair targeted therapy: the past or future of cancer treatment. Pharmacol Ther. 2016;160:65-83. doi:10.1016/j.pharmthera.2016. 02.003

11. Xu W, Wang S, Chen Q, et al. TXNL1-XRCC1 pathway regulates cisplatin-induced cell death and contributes to resistance in human gastric cancer. Cell Death Dis. 2014;13:e1055. doi:10.1038/cddis. 2014.27

12. Chen W, Wang J, Li X, et al. Prognostic significance of BRCA1 expression in gastric cancer. Med Oncol. 2013;30(1):423. doi:10. 1007/s12032-012-0423-5

13. Liu H. Research progress on Chinese materia medica for reversing tumor multidrug resistance. Chin Tradit Herbal Drugs. 2015;46 (7):1096-1102.

14. Li M, Zhang B, Zhang Z, et al. Stem cell-like circulating tumor cells indicate poor prognosis in gastric cancer. Biomed Res Int. 2014;2014:981261.

15. Lo Y, Liu Y, Ho Y-S. Reversing multidrug resistance in Caco-2 by silencing MDR1, MRP1, MRP2, and BCL-2/BCL-xL using liposomal antisense oligonucleotides. PLoS One. 2014;9(3):e90180. doi:10.1371/journal.pone.0090180

16. Brügger D, Brischwein $\mathrm{K}$, Liu C, et al. Induction of drug resistance and protein kinase $\mathrm{C}$ genes in A2780 ovarian cancer cells after incubation with antineoplastic agents at sublethal concentrations. Anticancer Res. 2002;22(6C):4229-4232.

17. Kwon HC, Roh MS, Oh SY, et al. Prognostic value of expression of ERCCl, thymidylate synthase, and glutathione S-transferase Pi for 5 -fluorouracil/oxaliplatin chemotherapy in advanced gastric cancer. Ann Oncol. 2007;18(3):504-509.

18. Yang L. The Study of c-MET Regulating Epithelial-Mesenchymal Transition in GC and Related Mechanisms in Acquired Resistance to Chemotherapy [dissertation]. Beijing: Medical School of Chinese PLA; 2017.
19. Zhang C. Preliminary Study on the Relationship Between Epithelial Mesenchymal Transformation (EMT) and Drug Resistance and Metastasis in Gastric Cancer [dissertation]. Yan'an: Yan'an University; 2012.

20. Lu M, Tao G, Fang Y, Shang Z, Ren L. Advances of hypoxia-induced chemotherapy resistance in gastric cancer. Chin J Clin Oncol. 2019;46(13):700-703.

21. Kewitz S, Kurch L, Volkmer I, Staege MS. Stimulation of the hypoxia pathway modulates chemotherapy resistance in Hodgkin's lymphoma cells. Tumor Biol. 2016;37(6):8229-8237. doi:10.1007/ s13277-015-4705-3

22. Lei $\mathrm{T}$, Liu L, Han $\mathrm{S}$, Guo $\mathrm{X}$, Ding J. The mechanism of hypoxia-induced multidrug resistance in gastric cancer. Chin J Gastroenterol Hepatol. 2011;20(5):414-418.

23. Xuan Y, Wang Y. Hypoxia/IL- $1 \alpha$ axis promotes gastric cancer progression and drug resistance. J Dig Dis. 2017;18(9):511-520. doi:10.1111/1751-2980.12496

24. Danza K, Silvestris N, Simone G, et al. Role of miR-27a, miR-181a and miR-20b in gastric cancer hypoxia-induced chemoresistance. Cancer Biol Ther. 2016;17(4):400-406. doi:10.1080/15384047.20 16.1139244

25. Magee P, Shi L, Garofalo M. Role of microRNAs in chemoresistance. Ann Transl Med. 2015;3(21):332.

26. Shi J, Mou X, Wang Y, et al. Advances in the study of microRNAs in the monitoring of drug resistance in gastric cancer. $J$ Qingdao Univ. 2018;54(6):740-746.

27. Li Y. Curcumin Reversed Multi-drug Resistance of Human Gastric Cancer Cells SGC7901/VCR in vitro [dissertation]. Xi'an: Fourth Military Medical University; 2006.

28. Feng Z. Reversal of Multidrug Resistance of Gastric Cancer Cell Line SGC7901/ADR by Curcumin and Verapami [dissertation]. Chongqing: Chongqing Medical Universtiy; 2012.

29. Kang Y, Hu W, Bai E, et al. Curcumin sensitizes human gastric cancer cells to 5-fluorouracil through inhibition of the NFKB survival-signaling pathway. Onco Targets Ther. 2016;9:7373-7384. doi:10.2147/OTT.S118272

30. Yu L, Wu J, Dai N, Yu H, Si J. Curcumin reverses chemoresistance of human gastric cancer cells by downregulating the NF- $\kappa B$ transcription factor. Oncol Rep. 2011;26(5):1197-1203.

31. Liu W, Yuan J, Yang L, Chang J. Mechanisms of curcumin to reverse herceptin resistance in gastric cancer cells. Acta Pharm Sin B. 2018;53(11):1817-1824.

32. Yang J, Li J, Jiang S. Research progress in the anti-tumor mechanism of action of tanshinone IIA. Chin J Inf TCM. 2015;22(7):128-130.

33. Liu Y, Ni J, Liu L, Li F, Yin X. Tanshinone IIA inhibits doxorubicin resistance in gastric cancer cells. Chin J Physiol. 2019;35(12): 2208-2214.

34. Xu Z, Chen L, Xiao Z, et al. Potentiation of the anticancer effect of doxorubicin in drug-resistant gastric cancer cells by tanshinone IIA. Phytomedicine. 2018;51:58-67. doi:10.1016/j.phymed.2018.05.012

35. Wang L, Wei P, Qin Z, et al. Experimental study on reversion effect of elemene on multidrug resistance of human gastric cancer cell line. J Chengdu Univ TCM. 2015;28(2):51-53.

36. Song H. Mechanism of Reversal of Invasion and Metastasis of Multidrug Resistant Gastric Cancer Cells by $\beta$-Elemene [dissertation]. Shenyang: China Medical University; 2019.

37. Zhang Y, Mu X, Li E, et al. The role of E3 ubiquitin ligase Cbl proteins in $\beta$-elemene reversing multi-drug resistance of human gastric adenocarcinoma cells. Int J Mol Sci. 2013;14(5):10075-10089. doi:10.3390/ijms140510075

38. Zhang Y, Song N, Liu Y, et al. Reversal of resistance to adriamycin in human gastric cancer by $\beta$-elemene. J Chin Med Univ. 2011;40 (11):968-993.

39. Yang Y, Chen Y, Bai J, et al. Effect of $\beta$-elemene on growth of multidrug-resistance gastric cancer cells xenografts in nude mice. Prog Anat Sci. 2019;25(3):229-231. 
40. Bai X, Liu Y, Song W, et al. Anticancer activity of tetrandrine by inducing pro-death apoptosis and autophagy in human gastric cancer cells. J Pharm Pharmacol. 2018;70(8):1048-1058. doi:10.1111/jphp. 12935

41. Li Y, Zhao Q, Tan B, et al. Effect and mechanisms of TET on human gastric carcinoma cell line SGC7901 and SGC7901/ADR. Chin J Integr Tradit West Med. 2014;34(1):66-70.

42. Zhang X. Effects of TET on the expression of ZNF139, MDR1 and GST- $\pi$ mRNA in multidrug resistant gastric cancer cells. $J$ Southeast Univ. 2018;37(3):467-470.

43. Guo X. Baicalein Inhibits the Invasion and Migration of Gastric Cancer BGC-823 Cells and Epithelial-Mesenchymal Transition Through NF-кB/Snail Signaling Pathway [dissertation]. Hangzhou: Zhejiang University; 2017.

44. Wang L. Baicalein Reverses Hypoxia-Induced Resistance to 5-Fluorouracil in AGS Cells Via Down-Regulating the Function of Drug Efflux Pump by HIF-la Signaling Pathway [dissertation] Fuzhou: Fujian Medical University; 2016.

45. Chen F, Zhuang M, Zhong C, et al. Baicalein reverses hypoxiainduced 5-FU resistance in gastric cancer AGS cells through suppression of glycolysis and the PTEN/Akt/HIF-1 $\alpha$ signaling pathway. Oncol Rep. 2015;33(1):457-463. doi:10.3892/or.2014. 3550

46. Hang Q. Inhibitory Effect of Baicalein on Gastric Cancer Resistant Cells SGC7901/DDP via the Modulation of Notch 1 and IncRNA AK022798 [dissertation]. Anhui: Anhui Medical University; 2014.

47. Zheng Z, Li C, Bo W. Effect and mechanism of arsenictrioxide $\left(\mathrm{AS}_{2} \mathrm{O}_{3}\right)$ on MRP mRNA expression on human Gastric carcinoma cells in vitro. J Mod Oncol. 2009;17(11):2082-2084.

48. Tang Y, Geng L, Bo W. Effect of arsenic trioxide on apoptosis and LRP expression on human gastric carcinoma cells. West China $J$ Pharm Sci. 2009;24(5):497-498.

49. Qi Q, Yu L, He X, Zhang B, Zhao Y. The effect of arsenic trioxide on reversal in cell model of multidrug-resistance gastric carcinoma. Med $J$ Qilu. 2014;29(5):404-410.

50. Zhao Y, Yu L, Liu B, He X, Zhang B. Downregulation of P-gp, Ras and $\mathrm{p}$-ERK1/2 contributes to the arsenic trioxide-induced reduction in drug resistance towards doxorubicin in gastric cancer cell lines. Mol Med Rep. 2015;12(5):7335-7343. doi:10.3892/mmr.2015.4367

51. Ye Q. Study on the Reversal Effect of Astragaloside IV Onmultidrug Resistance of the Gastric Cancer Cell SGC7901/DDP and its Mechanism [dissertation]. Nanjing: Nanjing University of Chinese Medicine; 2017.

52. Wang Y, Zou Y, Pei J. Reversal of resistance to trastuzumab in NCI-N87 tumor-bearing nude mice of aqueous extract of taxus Chinensis. Actc Chinese Medicine. 2019;34(255):1700-1704.

53. Li H, Chen Z, Liu M, Yang Y, Wang J, Wu H. Effect of target analysis of matrine on miRNA expression related to drug resistance in SGC7901/DDP cells of gastric cancer. Chin J Gerontol 2015;35:4746-4748.

54. Shi S, Zhang H. Reversal of multidrug resistance by neferine in human gastric carcinoma cell line SGC7901/VCR and its mechanism. Pract J Cancer. 2012;27(4):334-345.

55. Fang S, Zhu W, Zhang Y, Shu Y, Liu P. Paeoniflorin modulates multidrug resistance of a human gastric cancer cell line via the inhibition of NF-кB activation. Mol Med Rep. 2012;5(2):351-356.

56. Zhang Y, Tian X, Liu Y, et al. Effects of bufalin on proliferation and apoptosis of SGC7901/CDDP and its underlying mechanisms. J Xian Jiaotong Univ. 2012;33(4):498-514.

57. Zhao H, Zhao D, Jin $\mathrm{H}$, et al. Bufalin reverses intrinsic and acquired drug resistance to cisplatin through the AKT signaling pathway in gastric cancer cells. Mol Med Rep. 2016;14(2):1817-1822. doi:10. 3892/mmr.2016.5426

58. Li H, Li X, Cai D, Zhang Q, Liu J. Effect of oleanolic acid on the proliferation of cisplatin-resistant SGC-7901 cells and its mechanism. Chinese Pharmacol Bull. 2009;25(10):1334-1337.
59. Li H, Wang J, Qi Z, Zhou L, Xing G, Liu J. Effect of oleanolic acid on proliferation and apoptosis of cisplatin-resistant gastric cancer cell line SGC-7901. Chin J Gerontol. 2010;30(5):667-668.

60. Guo X, Liu J, Ma S, Zhang P, Sun W. Avicularin reversed multidrug-resistance in human gastric cancer through enhancing Bax and BOK expressions. Biomed Pharmacother. 2018;103:67-74. doi:10.1016/j.biopha.2018.03.110

61. Tang Q, Wang Y, Ma L, et al. Peiminine serves as an adriamycin chemosensitizer in gastric cancer by modulating the EGFR/FAK pathway. Oncol Rep. 2018;39(3):1299-1305.

62. Yang W, Lu S, Jia N, Zhao G. Effect of tetramethylpyrazine on proliferation and apoptosis and expressions of MDR1 and GST- $\pi$ in human gastric carcinoma cell line SGC-7901/ADR. Chin Arch Tradit Chin Med. 2015;33(8):2008-2010+2081-2082.

63. Deng W, Lv S, Wang Q, Lin Y, Duan C, Lu M. Reversal effect of sophoridine on B7 -H1 -mediated multidrug resistance in human gastric cancer cells. J New Chin Med. 2013;45(1):140-144.

64. Teng F, Xu Z, Lv H, et al. Triptolide reverses apatinib resistance in gastric cancer cell line MKN45 via inhibition of heat shock protein 70. Chin J Oncol. 2018;40(2):92-98.

65. Long L, Xu W, Shen H, Fang Y. Triptolide enhances the sensitivity of SGC7901/CDDP cells to cisplatin via inhibiting microRNA-21 expression. J Jiangsu Univ. 2012;22(3):213-217.

66. Wang T, Wei J, Qian X, Ding Y, Yu L, Liu B. Gambogic acid, a potent inhibitor of survivin, reverses docetaxel resistance in gastric cancer cells. Cancer Lett. 2008;262(2):214-222. doi:10.1016/j. canlet.2007.12.004

67. Chen Z, Wang P, Huang W, Liu L. Experimental study on effects of shengmai injection: enhancing 5-FU anti-tumor efficacy and reducing its toxicity. J Chin Integr Med. 2005;3(6):476-479.

68. Yan M. Study on Mechanism of Shengmai Injection Reversing Multidrug Resistance in Gastric Cancer Based on JNK Signaling Pathway [dissertation]. Shanxi: Shanxi Provincial Institute of TCM; 2017.

69. Liu L, Song W, Hao S, Wang X. Reversal effect of shengmai injection containing serum on multidrug resistant human gastric cancer cell and its possible mechanism. Chin J Tradit Chin Med Pharm. 2018;33(9):4204-4208.

70. Shan S, Xiao T, Chen J, et al. Kanglaite attenuates UVB-induced down-regulation of aquaporin-3 in cultured skin nocytes. Int $\mathrm{J} \mathrm{Mol}$ Med. 2012;29(4):625-629. doi:10.3892/ijmm.2011.873

71. Zhan Y, Huang X, Cao J, et al. Clinical safety and efficacy of kanglaite $^{\circledR}$ (Coix Seed Oil) injection combined with chemotherapy in treating patients with gastric cancer. Asian Pac J Cancer Prev. 2012;13(10):5319-5321. doi:10.7314/APJCP.2012.13.10.5 319

72. Zhang X, Liu L, Zhang X, Bo P. Kanglaite inhibits the expression of drug resistance genes through suppressing PVT1 in cisplatin-resistant gastric cancer cells. Exp Ther Med. 2017;14(2):1789-1794. doi:10.38 92/etm. 2017.4650

73. Shu P, Tang H, Zhou B, et al. Effect of Yiqi Huayu Jiedu decoction on stages II and III gastric cancer: a multicenter, prospective, cohort study. Medicine. 2019;98(47):e17875. doi:10.1097/MD.0000000000 017875

74. Yang Q. The Expression influence of MDR1 Gene Expression about Jianpiyangwei Decoction on Human Gastric Carcinoma Multidrug Resistance Cell Line BGC823/5-Fu and the Exploration of Possible Cell Signal Pathway [dissertation]. Nanjing: Nanjing University of Chinese Medicine; 2013.

75. Tang H. The Influence of Differentiation about Drug-Containing Serum of Jianpi Yangwei Decoction on CD44(+) Gastric Cancer Stem Cells [dissertation]. Nanjing: Nanjing University of Chinese Medicine; 2016.

76. Fu Y. The Influence of Drug Resistance about Jian pi yang wei Decoction on CD44(+) Gastric Cancer Stem Cells [dissertation]. Nanjing: Nanjing University of Chinese Medicine; 2017. 
77. Li W, Li Y, Yu C, He Y. Reversal of multidrug resistance by the Chinese medicine Yiqi Jianpi Huaji Decoction and the mechanism of action in human gastric cancer SGC7901/ VCR cells. Evid Based Complement Alternat Med. 2015;20 15:390812.

78. Xia Y, Wang J, Gao C. Influence of shenghe powder with chemotherapy on recurrence and metastatic rate of post-operative gastric cancer. Chin J Surg Integr Tradit West Med. 1996;2 (6):402-404.

79. Wang J, Xia Y, Wang H, Hou Z. Chinese herbs of shenghe powder reverse multidrug resistance of gastric carcinoma SGC-7901. Integr Cancer Ther. 2007;6(4):400-404. doi:10.1177/1534735407 309765

80. Li C, Li M, Hou P. Effect of combination of modern medicine with traditional Chinese medicine on the expression of gene LRP and MRP of cells line SGC -7901/ADR in human gastric cancer. J Baotou Med College. 2013;29(4):8-10.
81. Li M, Li C, Hou P. Combination of modern medicine and traditional Chinese medicine effect on obtains control gene Bcl-2, Bax mRNA of human gastric cancer cells line SGC-7901/ADR. Chin Foreign Med Treat. 2013;7:11-13.

82. Fu G, Zhang A. Banzhen 1, tetra methylpyrazine and 5-fluorouracil effect on human gastric cancer cells glutathione-S-transferase. Chin Med Pharm. 2012;2(4):34-35.

83. Xu J, Fan Z, Sun J, et al. Effects of "Chang Wei Qing” in treating advanced gastrointestinal cancer and its effects on peripheral blood MDR1 mRNA. Shanghai J Tradit Chin Med. 2007;41(5):40-42.

84. Hao S, Liu L, Li Y. The effect of ertengsanjie on multi-drug resistance of SGC7901/VCR cells and the relationship with P-gp. J Basic Clin Oncolo. 2010;23:480-482.

85. Xu L, Wang M, Xu D, Zhou C. Experimental study on modified "sanwubai powder" in affecting muti-drug resistance gene expression of tumor. Shanghai J Tradit Chin Med. 2005;39(8):59-60.

\section{Publish your work in this journal}

Cancer Management and Research is an international, peer-reviewed open access journal focusing on cancer research and the optimal use of preventative and integrated treatment interventions to achieve improved outcomes, enhanced survival and quality of life for the cancer patient.
The manuscript management system is completely online and includes a very quick and fair peer-review system, which is all easy to use. Visit http://www.dovepress.com/testimonials.php to read real quotes from published authors. 